\title{
A Novel Four-port-sharing Approach for Concomitant Cholecystectomy With Laparoscopic Sleeve Gastrectomy
}

\section{Fang-Chin Hsu}

Tri-Service General Hospital

Hai-Ning Hsu

Tri-Service General Hospital

Hsin-Mei Pan

Tri-Service General Hospital

Wan-Ting Hung

Tri-Service General Hospital

Guo-Shiou Liao

Tri-Service General Hospital

\section{Teng-Wei Chen}

Tri-Service General Hospital

\section{De-Chuan Chan}

Tri-Service General Hospital

Kuo-Feng Hsu ( $\sim$ hsukf97@ndmctsgh.edu.tw )

Tri-Service General Hospital

\section{Research Article}

Keywords: Bariatric surgery, concomitant, cholecystectomy, gastrectomy, port-sharing

Posted Date: September 29th, 2021

DOI: https://doi.org/10.21203/rs.3.rs-892287/v1

License: (1) (1) This work is licensed under a Creative Commons Attribution 4.0 International License. Read Full License 


\section{Abstract}

\section{Background}

Gallbladder disease is very common in obese patients. Concomitant cholecystectomy with laparoscopic sleeve gastrectomy (CC-LSG) may be necessary in such cases, and it has been proven safe when indicated. Herein, we developed a novel four-port-sharing technique for CC-LSG that can substitute the conventional trocar placement (6-7 ports).

\section{Methods}

A retrospective cohort study was conducted between January 2017 and March 2021 using a prospective database. Out of 159 patients who underwent LSG, 10 also received CC-LSG. The patients' demographic characteristics, intraoperative outcomes, and postoperative outcomes were examined.

\section{Results}

Of a total of 159 patients, 18 had cholelithiasis (11.3\%). Eight symptomatic and 2 asymptomatic patients (6 men and 4 women) with cholelithiasis underwent CC-LSG. No postoperative complications, including hemorrhage, bile leakage, staple leakage, incisional hernia, and wound infection, were noted.

\section{Conclusions}

In CC-LSG, the application of a four-port-sharing technique is safe and feasible, and it provides satisfying cosmetic outcomes for obese patients with symptomatic or asymptomatic cholelithiasis.

\section{Key Points}

- Concomitant cholecystectomy with laparoscopic sleeve gastrectomy (CC-LSG) has been proven safe when indicated.

- We propose a practical and novel four-port-sharing approach instead of the conventional trocar placement (6-7 ports) for CC-LSG.

- This four-port-sharing technique is a safe and feasible method and provides satisfying cosmetic results.

\section{Background}

Gallbladder disease is a common obesity-related illness. Rapid weight loss in obese patients undergoing bariatric surgery has been associated with a high incidence of gallstone formation [1]. Hence, concomitant cholecystectomy is routinely performed in all patients at the time of open bariatric surgery, before the application of laparoscopy [2]. Because laparoscopic techniques are being developed to perform bariatric surgery, the necessity of concomitant laparoscopic cholecystectomy (LC) has been discussed and been proven safe when indicated [3]. However, details regarding the surgical techniques 
for concomitant cholecystectomy and laparoscopic sleeve gastrectomy (CC-LSG) are rarely mentioned. Herein, we developed a novel four-port-sharing technique for CC-LSG.

\section{Methods}

A retrospective cohort study was conducted from our prospective database between January 2017 and March 2021. We included 159 patients who underwent LSG and 21 patients with gallbladder diseases, of whom 3 patients underwent LC before LSG due to biliary colic. The remaining 18 patients had cholelithiasis, including 10 patients who underwent concomitant LC with LSG and 8 asymptomatic patients who refused surgical interventions. The study was approved by the Ethical Committee. All procedures performed in the included studies that involved human participants were in accordance with the ethical standards of the institutional and/or national research committee and with the $1964 \mathrm{Helsinki}$ declaration and its later amendments or comparable ethical standards. Informed consent was obtained from all individual participants included in the study.

Patients were placed in the supine position under general anesthesia, and their skin was prepared. Four trocars were placed: one 12-mm trocar at the umbilicus, one 10-mm trocar along the left anterior-axillary line of the left lower abdomen, one 5-mm trocar along the right midclavicular line of the right upper abdomen, and one 5-mm trocar at the subxiphoid area of the epigastrium. Insufflation with carbon dioxide was performed at $12 \mathrm{~mm} \mathrm{Hg}$ pressure. A 10-mm, 30 extended length laparoscope was inserted through the umbilical trocar, a grasper was inserted through the right upper abdominal trocar, and a hook or a dissector was inserted through the subxiphoid trocar (Fig. 1a). The left upper abdominal trocar was inserted with a grasper and was used flexibly to enable adequate exposure of the surgical field (Fig. 1b). The LC procedure was then performed. After both the cystic artery and duct were safely divided, the gallbladder was removed from the liver bed and was put in a plastic bag. The second part of the operation involved LSG. A laparoscope was shifted to the left lower abdominal trocar, and we placed a LigaSure or an Endo-GIA (Medtronic, Minneapolis, Minnesota, US) for gastric stapling through the umbilical trocar. A liver retractor was inserted through the subxiphoid trocar, but in certain cases, the thread method (without the use of a trocar) was also applied for liver retraction. The grasper remained in the right upper abdominal trocar, and we used the same ports to perform LSG without any additional trocars (Fig. 2a \& b). Following the completion of gastric stapling, the gallbladder and the resected stomach were retrieved from the umbilical trocar.

Patients' characteristics such as age, gender, body weight, body height, and body mass index; the American Society of Anesthesiologists classification; comorbidities; operation time for LC and LSG; blood loss; drain placement; postoperative complications; and postoperative hospital stay were then analyzed.

\section{Results}

Of a total of 159 patients treated, 18 had cholelithiasis (11.3\%). Ten of these 18 patients subsequently underwent CC-LSG, including 8 symptomatic and 2 asymptomatic patients. The remaining 8 patients had 
asymptomatic cholelithiasis and refused concomitant $L C$ after discussion with the physicians. The mean age of patients that underwent CC-LSG was 36.5 years, with 6 males $(60.0 \%)$ and 4 females $(40.0 \%)$. The mean body weight and BMI were $118.7 \mathrm{~kg}$ and $42.8 \mathrm{~kg} / \mathrm{m}^{2}$, respectively. Three patients had type 2 diabetes mellitus (T2DM), 10 patients had hypertension, 7 patients had hyperlipidemia, and 4 patients had moderate to severe obstructive sleep apnea. Other baseline characteristics are summarized in Table 1. 
Characteristics of the patients undergoing concomitant cholecystectomy with laparoscopic sleeve gastrectomy

\begin{tabular}{ll} 
Variables & Patients (n=10) \\
Age, year & $36.5 \pm 11.5$ \\
\hline Gender (male/female) & $6 / 4$ \\
\hline Body weight, kg & $118.7 \pm 13.6$ \\
\hline Body height, m & $1.66 \pm 0.083$ \\
\hline Body mass index, kg/m ${ }^{2}$ & $42.8 \pm 3.6$ \\
\hline ASA classification & \\
\hline Class I & $3(30 \%)$ \\
\hline Class II & $5(50 \%)$ \\
\hline Class III & $2(20 \%)$ \\
\hline Comorbidities & \\
\hline T2DM & $3(30 \%)$ \\
\hline Hypertension & $10(100 \%)$ \\
\hline Hyperlipidemia & $7(70 \%)$ \\
\hline Moderate/Severe obstructive sleep apnea & $4(40 \%)$ \\
\hline Operation time for LC, minute & $50.5 \pm 4.4$ \\
\hline Operation time for LSG, minute & $109.8 \pm 12.6$ \\
\hline Blood loss, ml & $11 \pm 3.9$ \\
\hline Drain placement (present) & $10(100 \%)$
\end{tabular}

Postoperative complications

Hemorrhage

0

Bile leakage

0

Staple leakage

0

Wound infection

0

Postoperative hospital stay (day)

$4 \pm 1.1$

Data are presented as mean \pm standard deviation, $n$ (\%); ASA: American Society of Anesthesiologists; T2DM: type 2 diabetes mellitus; LC: laparoscopic cholecystectomy; LSG: laparoscopic sleeve gastrectomy 
The experienced surgeons performed laparoscopic surgery to all 10 patients, and no perioperative complications or conversions to open surgery were noted. The mean operation times were $50.5 \mathrm{~min}$ for LC and $109.8 \mathrm{~min}$ for LSG. Blood loss during the operation was $11 \mathrm{~mL}$. A drain was routinely placed to the left abdominal trocar site postoperatively. We did not register any postoperative complications, including hemorrhage, bile leakage, staple leakage, incisional hernia, and wound infection. All patients were discharged within 5 days post operation and remained in a stable condition during a 1-year followup.

\section{Discussion}

In a retrospective study with 21,137 patients who underwent LSG in the USA, only $2 \%$ received concomitant cholecystectomy [4]. In addition, an increased operative time with a higher risk of bleeding and pneumonia was noted in patients who underwent CC-LSG compared to patients who received LSG alone [4, 5]. However, CC-LSG was still considered a safe approach in symptomatic patients and was not found to increase hospital stay and complication rates [3-5].

By contrast, performing CC-LSG to obese patients with asymptomatic gallbladder stones still remains under debate. Raziel et al. demonstrated that $9.3 \%$ of patients with asymptomatic gallstones required cholecystectomy during the first postoperative year compared to only $2.7 \%$ of patients with normal preoperative gallbladders [6]. Furthermore, Altieri et al. reported that the LC rates after LSG were approximately $10 \%$, and a subsequent LC may not only be a more difficult procedure but it may also facilitate higher rates of common bile duct injury [7]. Another study reported that patients with asymptomatic gallstones had a similar risk of becoming symptomatic as healthy population [8]. In our study, a total of 10 patients had asymptomatic cholelithiasis, and only 2 patients underwent CC-LSG due to multiple gallstones. Patients without gallbladder disease refused concomitant LC. There is no postoperative biliary-related complication. In our practice, CC-LSG is a feasible and safe procedure when indicated.

Additional ports may be required to perform simultaneous operations in laparoscopic surgery. In general, 6 to 7 ports are needed to accomplish concomitant laparoscopic operations $[9,10]$. Ohta et al. proposed a total of 7 trocars in a port-sharing technique for LC and LSG, with 3 trocars being greater than $10 \mathrm{~mm}$ [11]. One additional trocar was placed in the right lateral abdomen for LC and 1 in the left abdomen for LSG. In our study, 4 trocars were considered an adequate number to perform CC-LSG. There are only 2 trocars equal to or larger than $10 \mathrm{~mm}$, including the umbilical trocar $(12 \mathrm{~mm})$ and the left abdominal trocar $(10 \mathrm{~mm})$. The 2 trocars are placed in the umbilicus and the left lower abdomen, respectively, to substitute the conventional sites of the upper abdomen. The advantages of changing the trocar sites include better cosmetic outcomes and a more comprehensive surgical filed, while longer devices may be necessary to reach the surgical site. Moreover, there was no incisional hernia noted during postoperative follow-up. In LSG, we shifted the laparoscope to the left abdominal trocar, and we placed the gastric 
stapling device in the umbilical trocar. This approach provides a clear field of view during posterior mobilization and gastric stapling. The remaining 2 trocars that we used were both $5 \mathrm{~mm}$ in size. In general approach three 12-mm trocars and three to four 5-mm trocars were used for LC and LSG. The four-port-sharing technique not only reduces the number of trocar incisions by reusing the ports, but it also decreases the size of trocars. Furthermore, this approach offers satisfying cosmetic results, and it may reduce postoperative pain compared to the general method that is currently used.

\section{Conclusion}

Although CC-LSG was not commonly performed in all bariatric surgeries, it is an approach that should be considered in patients with gallbladder disease. CC-LSG using a four-port-sharing technique can decrease the number and size of the trocars used, and it can provide better cosmetic results and less postoperative pain. Conclusively, the four-port-sharing technique is a safe and feasible option for obese patients undergoing CC-LSG.

\section{Abbreviations}

1. LC: laparoscopic cholecystectomy

2. CC-LSG: concomitant cholecystectomy and laparoscopic sleeve gastrectomy

3. T2DM: type 2 diabetes mellitus

\section{Declarations}

\section{Availability of data and materials}

All data generated or analysed during this study are included in this published article.

\section{Acknowledgements}

None.

\section{Funding}

None.

\section{Authors' Contributions}

All authors have read and approved the manuscript. $\mathrm{FCH}, \mathrm{HNH}$ : Study design, imaging analysis, drafting and editing the manuscript. HMP, WTH, GSL, TWC, DCC: imaging analysis and editing the manuscript. $\mathrm{KFH}$ : Study design, interpretation of data, supervising the study, and drafting the manuscript.

\section{Ethics declarations}


Ethics approval and consent to participate

The present study was approved by the Ethics Committee of Tri-Service General Hospital (\#B202105089).

The research reported in this paper was in compliance with the Helsinki Declaration. We obtained written informed consent from all patients.

Consent for publication

Not applicable.

Competing interest

The authors declare no competing interests.

\section{References}

1. Wudel LJ, Jr., Wright JK, Debelak JP, Allos TM, Shyr Y, Chapman WC: Prevention of gallstone formation in morbidly obese patients undergoing rapid weight loss: results of a randomized controlled pilot study. J Surg Res 2002, 102(1):50-56.

2. Fobi M, Lee H, Igwe D, Felahy B, James E, Stanczyk M, Fobi N: Prophylactic cholecystectomy with gastric bypass operation: incidence of gallbladder disease. Obes Surg 2002, 12(3):350-353.

3. Wood SG, Kumar SB, Dewey E, Lin MY, Carter JT: Safety of concomitant cholecystectomy with laparoscopic sleeve gastrectomy and gastric bypass: a MBSAQIP analysis. Surg Obes Relat Dis 2019, 15(6):864-870.

4. Dakour-Aridi HN, El-Rayess HM, Abou-Abbass H, Abu-Gheida I, Habib RH, Safadi BY: Safety of concomitant cholecystectomy at the time of laparoscopic sleeve gastrectomy: analysis of the American College of Surgeons National Surgical Quality Improvement Program database. Surg Obes Relat Dis 2017, 13(6):934-941.

5. Coşkun H, Hasbahçeci M, Bozkurt S, Çipe G, Malya F, Memmi N, Karatepe O, Akçakaya A, Müslümanoğlu M: Is concomitant cholecystectomy with laparoscopic sleeve gastrectomy safe? Turk J Gastroentero/ 2014, 25(6):624-627.

6. Raziel A, Sakran N, Szold A, Goitein D: Concomitant cholecystectomy during laparoscopic sleeve gastrectomy. Surg Endosc 2015, 29(9):2789-2793.

7. Altieri MS, Yang J, Nie L, Docimo S, Talamini M, Pryor AD: Incidence of cholecystectomy after bariatric surgery. Surg Obes Relat Dis 2018, 14(7):992-996.

8. Yardimci S, Coskun M, Demircioglu S, Erdim A, Cingi A: Is Concomitant Cholecystectomy Necessary for Asymptomatic Cholelithiasis During Laparoscopic Sleeve Gastrectomy? Obes Surg 2018, 28(2):469-473.

9. Schizas D, Katsaros I, Karatza E, Kykalos S, Spartalis E, Tsourouflis G, Dimitroulis D, Nikiteas N: Concomitant Laparoscopic Splenectomy and Cholecystectomy: A Systematic Review of the 
Literature. J Laparoendosc Adv Surg Tech A 2020, 30(7):730-736.

10. Hiratsuka T, Ohta M, Sonoda K, Yamamura S, Nishizaki T, Matsusaka T, Kitano S: Simultaneous operation of laparoscopy-assisted distal gastrectomy with laparoscopic cholecystectomy. Hepatogastroenterology 2007, 54(78):1645-1647.

11. Ohta M, Tada K, Endo Y, Nakanuma H, Fujinaga A, Kawamura M, Masuda T, Kawasaki T, Watanabe K, Hirashita $\mathrm{T}$ et al: Port-sharing techniques for laparoscopic cholecystectomy and sleeve gastrectomy. Surg Today 2021.

\section{Figures}

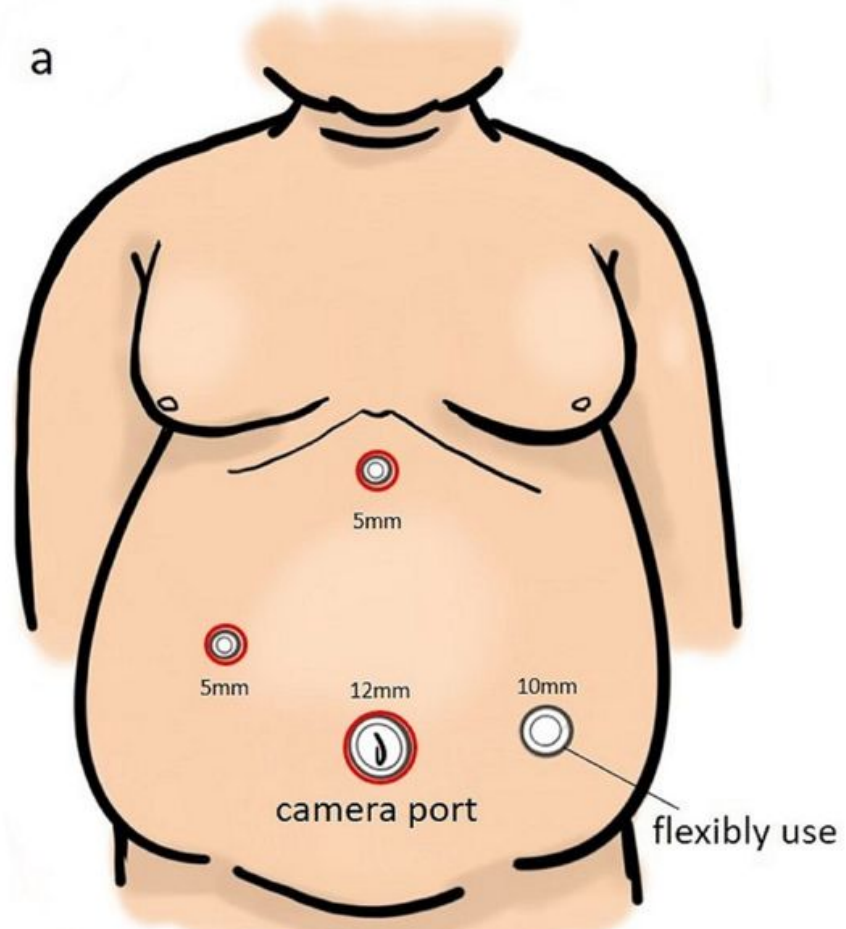

b

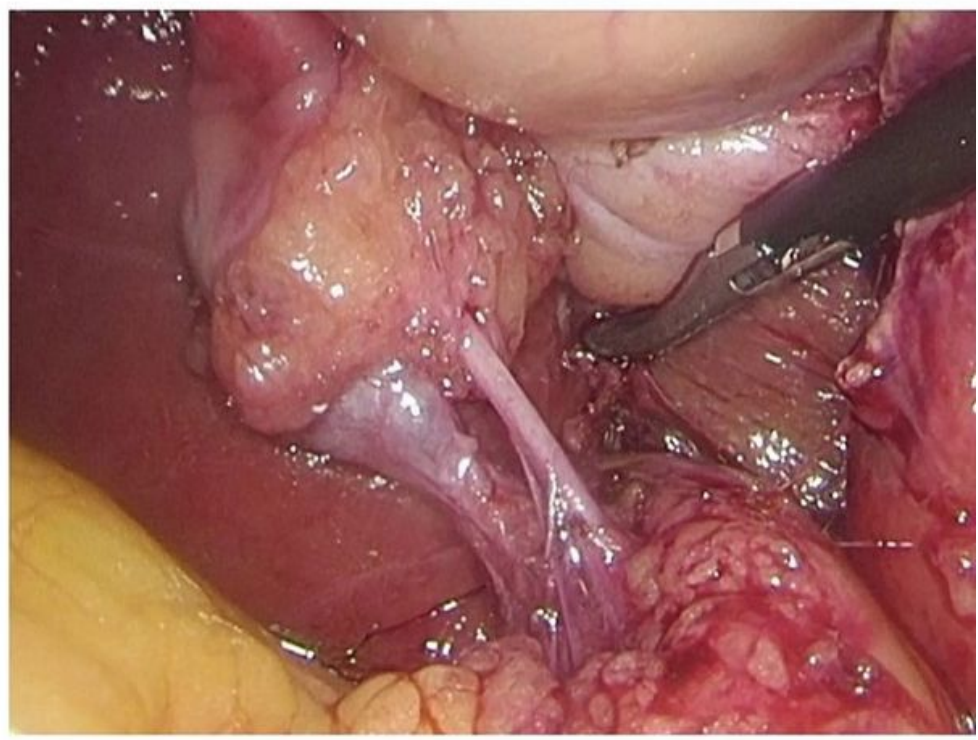

Figure 1

Trocar placement when performing LC (a) A laparoscope was inserted through the umbilical trocar (12 $\mathrm{mm})$, a grasper was inserted through the right upper abdominal trocar $(5 \mathrm{~mm})$, and a hook or a dissector was inserted through the subxiphoid trocar $(5 \mathrm{~mm})$. In total, 3 trocars (indicated by the red circles) were routinely used. The left abdominal trocar was used flexibly to enable adequate exposure of the surgical field. (b) The surgical view of Calot's triangle was well exposed. 


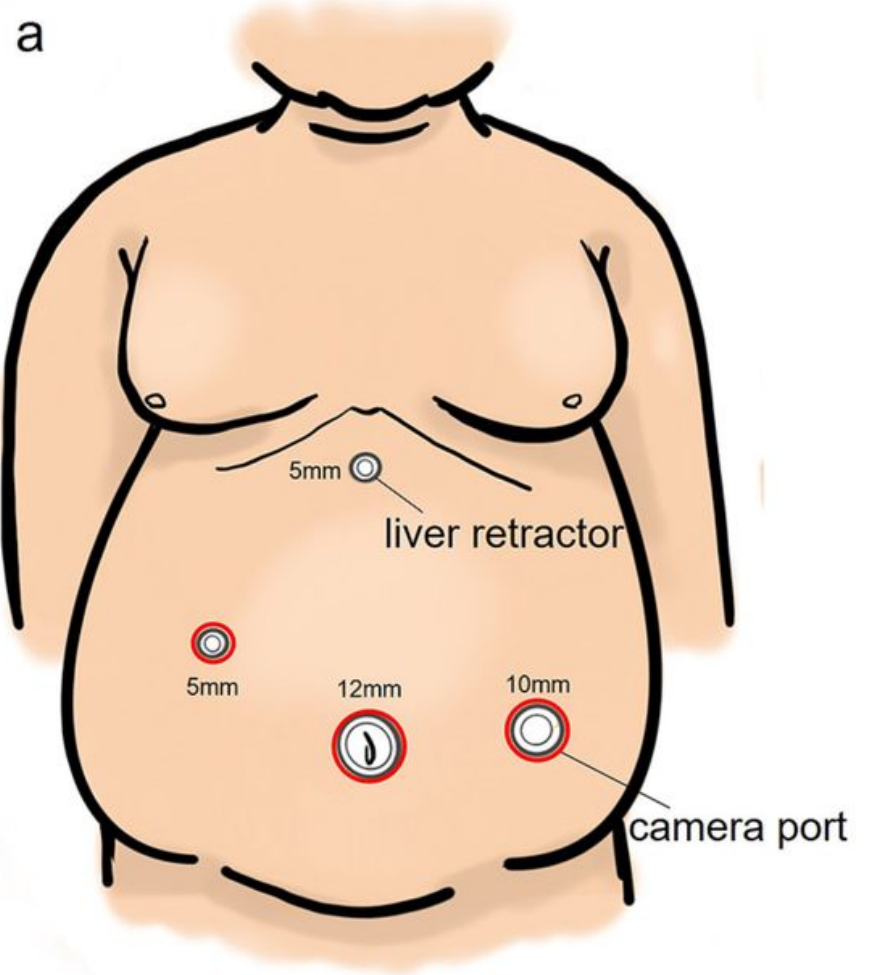

b

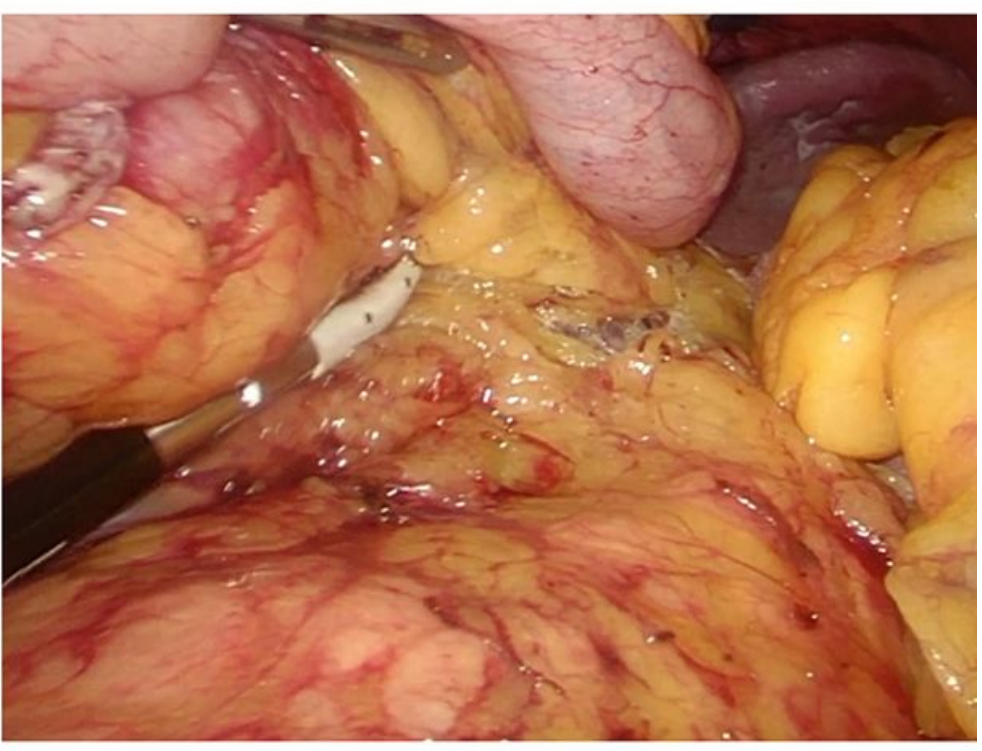

Figure 2

Trocar placement when performing LSG (a) A laparoscope was shifted to the left abdominal trocar (10 $\mathrm{mm})$. A LigaSure or an Endo GIA for gastric stapling was inserted through the umbilical trocar $(12 \mathrm{~mm})$, and a grasper remained in the right upper abdominal trocar $(5 \mathrm{~mm})$. In total, 3 trocars (indicated by the red circles) were routinely used. A liver retractor was inserted through the subxiphoid trocar or the thread method (without trocar use) was employed for liver retraction. (b) Surgical view of posterior mobilization during SG. 